Technological University Dublin

DÜBLIN

ARROW@TU Dublin

2005-01-01

\title{
Asymptotic methods in the spectral analysis of Sturm-Liouville operators
}

Daphne Gilbert

Technological University Dublin, Daphne.Gilbert@tudublin.ie

Follow this and additional works at: https://arrow.tudublin.ie/scschmatart

Part of the Mathematics Commons

\section{Recommended Citation}

Gilbert, D. (2005). Asymptotic methods in the spectral analysis of Sturm-Liouville operators. SturmLiouville theory: Past and Present, pg. 121-136. doi:10.1007/3-7643-7359-8_6

This Article is brought to you for free and open access by the School of Mathematics at ARROW@TU Dublin. It has been accepted for inclusion in Articles by an authorized administrator of ARROW@TU Dublin. For more information, please contact arrow.admin@tudublin.ie, aisling.coyne@tudublin.ie,gerard.connolly@tudublin.ie.

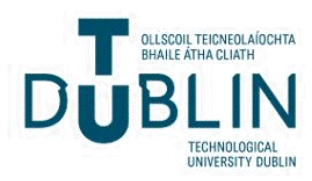




\title{
Asymptotic Methods in the Spectral Analysis of Sturm-Liouville Operators
}

\author{
Daphne Gilbert \\ School of Mathematical Sciences, Dublin Institute of Technology, \\ Kevin Street, Dublin 8, Ireland \\ daphne.gilbert@dit.ie
}

\begin{abstract}
We consider the relationship between the asymptotic behaviour of solutions of the singular Sturm-Liouville equation and spectral properties of the corresponding selfadjoint operators. In particular, we review the main features of the theory of subordinacy by considering two standard cases, the half-line operator on $L_{2}([0, \infty))$ and the full-line operator on $L_{2}(\mathbf{R})$. It is assumed that the coefficient function $q$ is locally integrable, that 0 is a regular endpoint in the half-line case, and that Weyl's limit point case holds at the infinite endpoints. We note some consequences of the theory for the well-known informal characterisation of the spectrum in terms of bounded solutions. We also consider extensions of the theory to related differential and difference operators, and discuss its application, in conjunction with other asymptotic methods, to some typical problems in spectral analysis.
\end{abstract}

\section{Introduction}

In its original formulation, the Sturm-Liouville boundary value problem consists of a linear second order ordinary differential equation expressible in the form

$$
L u:=-u^{\prime \prime}(r)+q(r) u(r)=\lambda u(r), \quad r \in I \subseteq \mathbf{R}, \quad q: I \rightarrow \mathbf{R}, \quad \lambda \in \mathbf{C},
$$

together with suitable separated or periodic boundary conditions at the endpoints of a finite interval $I$. The spectrum of the associated selfadjoint operator consists of an increasing sequence of isolated real eigenvalues accumulating at infinity, the corresponding eigenfunctions being non-trivial solutions of (1) which satisfy the endpoint conditions [5], [12], [21], [31], [47]. Extension to the case where one of the endpoints of $I$ is singular was achieved by Weyl in 1910 [51], and if $I=[0, \infty)$ or $(-\infty, \infty)$, then (1) is often referred to as the one-dimensional time independent Schrödinger equation, following subsequent 
recognition of its importance in the mathematical description of quantum phenomena (see eg [39]). If a singular endpoint is in Weyl's limit point case then essential spectrum, which can itself contain both discrete and continuous parts, may also or alternatively be present, and while the (generalised) eigenfunctions are still solutions of the Sturm-Liouville equation (1), their behaviour is now more subtle.

Recognition of the close relationship between solutions of the differential equation and spectral properties was already evident in the 1836 paper of Sturm, where the link between the number of points of the spectrum below an eigenvalue and the number of zeros in the associated eigenfunction was noted [47]. Analogues of such properties for the singular case, as well as numerous further connections between solutions and spectra, were identified in the mid-twentieth century by Hartman, Wintner and others (see e.g. [18], [20], [52]), while contemporary investigations in the Soviet Union contributed a number of independent results in this regard [16, Chapter V]. In more recent work linking polynomially bounded solutions to spectral properties, techniques which are applicable to both one-dimensional and higher dimensional problems have been developed (see e.g. [22], [40]).

Different challenges emerged in the late 1950's with the development of rigorous scattering theory and a corresponding awareness of the importance of distinguishing the absolutely continuous component from other parts of the essential spectrum, in connection with existence and completeness of the wave operators [1], [2], [25]. Subsequent efforts to identify distinguishing features of the absolutely continuous eigenfunctions include work by Carmona [6] and Weidmann [48], [50], the method of subordinacy, to be outlined in Section 3 [15], [13], [14], [35], and the use of transfer matrices by Last and Simon [30].

Prior to a seminal paper of Pearson [34], showing that apparently innocuous potentials can give rise to purely singular continuous spectrum on $\mathbf{R}^{+}$, significant activity was focussed on seeking conditions under which the absence of singular continuous spectrum could be assured (see e.g. [36, Chapter XIII]). The subsequent realisation that singular continuous spectrum is generically present in a variety of situations [9] has stimulated further research activity in recent years (see e.g. [29], [41]), while the method of subordinacy and its extensions provide some insight into the hitherto obscure behaviour of the associated eigenfunctions [15], [23].

The principal focus of this paper is an overview of the method of subordinacy and its extensions, together with a brief discussion of the wider historical context and some illustrative examples to demonstrate its role in applications. Details of the derivation of the theory and of related background results can be found in the cited references. 


\section{Bounded Solutions and Spectral Properties}

The usefulness in practice of methods which characterise the spectrum in terms of the asymptotic behaviour of solutions is well known, and in this section we briefly discuss two such approaches, both of which have informed and are informed by the method of subordinacy. To fix ideas, we restrict attention to the half-line case where $I=[0, \infty), L$ is regular at 0 and in Weyl's limit point case at infinity, and $q$ is locally integrable. In this case it is known that an initial condition of the form

$$
\cos (\alpha) u(0)+\sin (\alpha) u^{\prime}(0)=0, \quad \alpha \in[0, \pi),
$$

is needed at the origin to render the associated operator $H_{\alpha}$ selfadjoint and that, under the Hilbert space formulation outlined in Section 3, no endpoint condition at infinity is required. The spectrum, $\sigma\left(H_{\alpha}\right)$, is then the complement in $\mathbf{C}$ of the set of all $\lambda$ for which the resolvent operator $\left(H_{\alpha}-\lambda I\right)^{-1}$ is bounded and everywhere defined; an equivalent definition can also be formulated in terms of the corresponding spectral function, $\rho_{\alpha}$ (see Section 3.1).

It follows from the classical separation and comparison theorems of Sturm (see [21], [42]) that the real line may be partitioned into oscillatory and nonoscillatory regions, separated by a so-called parabolic point $\lambda^{\star} \in \mathbf{R} \cup\{ \pm \infty\}$. For $\lambda \in\left(-\infty, \lambda^{\star}\right)$ any, and hence all, solutions of (1) have a finite number of zeros for $r \geq 0$, while for $\lambda>\lambda^{\star}$ any, and hence all, solutions of (1) have a countably infinite number of zeros accumulating only at infinity; we therefore refer to (1) as being non-oscillatory or oscillatory according as $\lambda \in\left(-\infty, \lambda^{\star}\right)$ or $\lambda \in\left(\lambda^{\star}, \infty\right)$ respectively [20].

Since (1) is oscillatory at $\lambda$ if and only if the spectrum on $(-\infty, \lambda)$ is an infinite set [18], the spectrum of $H_{\alpha}$ on $\left(-\infty, \lambda^{\star}\right)$ consists of isolated eigenvalues only, possibly accumulating at $\lambda^{\star}$, with every $\lambda \in\left(-\infty, \lambda^{\star}\right)$ being an eigenvalue for some value of $\alpha$ in (2) [19]. It follows that there exists an $L_{2}([0, \infty))$ solution $u_{\lambda}(r)$ of (1) for each $\lambda<\lambda^{\star}$; this is known as a principal solution and satisfies

$$
\lim _{r \rightarrow \infty} \frac{u_{\lambda}(r)}{v_{\lambda}(r)}=0
$$

whenever $v_{\lambda}(r)$ is a linearly independent solution of the same equation, with $u_{\lambda}(r)=o(1)$ as $r \rightarrow \infty$ if $\lambda^{\star}<\infty$ [20], [52]. The restriction of the spectrum to $\left(-\infty, \lambda^{\star}\right)$ is then given by

$$
\begin{aligned}
\sigma\left(H_{\alpha}\right) \cap\left(-\infty, \lambda^{\star}\right)= & \left\{\lambda<\lambda^{\star}:\right. \text { there exists a principal solution } \\
& \text { of }(1) \text { satisfying the boundary condition }(2)\} .
\end{aligned}
$$

A second and more pervasive approach to the spectral analysis of the singular problem, particularly among physicists, effectively bypasses the intricacies of 
Hilbert space theory by defining the "spectrum", $S_{\alpha}$, to be the set of all $\lambda$ for which a non-trivial solution $u(r)$ of (1) and (2) satisfies the further condition:

$$
u(r)=O(1) \text { as } r \rightarrow \infty .
$$

In the case where $\alpha=0, q \equiv 0$, this yields $S_{\alpha}=(0, \infty)$, which is not a closed set, so that $S_{\alpha} \neq \sigma\left(H_{\alpha}\right)$ in general; however, since 0 is not an eigenvalue of $H_{\alpha}$, and $\sigma\left(H_{\alpha}\right)=[0, \infty)$, we see that $S_{\alpha}=\sigma\left(H_{\alpha}\right)$ except for a set which has measure zero with respect to both Lebesgue and spectral measures.

The longstanding conjecture that $S_{\alpha}=\sigma\left(H_{\alpha}\right)$ a.e. holds in general appears to be still unresolved so far as the essental spectrum is concerned (see e.g. [16, Chapter V], [40, Section C5]), although study of related discrete operators with almost periodic potentials suggests that for this part of the spectrum the conjecture is false [53]. In the case of isolated point spectrum, the conjecture was refuted for $\lambda^{\star}=\infty$ by a counterexample due to Hartman and Wintner, in which for each $\lambda \in \mathbf{R}$, the square integrable solution fails to be $O(1)$ as $r \rightarrow \infty$ (see [20, (v)(c) and p. 648]); we then have $S_{\alpha}=\emptyset$, while $\sigma\left(H_{\alpha}\right)$ consists of an infinite sequence of isolated eigenvalues accumulating at infinity. However, the conjecture is confirmed for isolated point spectrum when $\lambda^{\star}<\infty$, since if $\lambda$ is not in the essential spectrum in this case, the $L_{2}([0, \infty))$ solution of (1) is $O\left(r^{-N}\right)$ as $r \rightarrow \infty$ for every fixed $N$ [52].

This informal or "working" definition of the spectrum in terms of bounded solutions seems to have originated from concern that solutions of the wave equation of quantum mechanics (i.e. the time dependent Schrödinger equation) be "physically admissible", taking into account the accepted interpretation of the square of the modulus of the value of the wave function at a point as the position probability density (see e.g. [10, Section 38], [38, Chapter II]). Apart from its apparent agreement with physical intuition, the working definition is undoubtedly attractive in that it is conceptually simple, easy to apply and agrees with the Hilbert space definition of the spectrum (at least up to null sets) in many familiar elementary examples. We shall return to the relationship between bounded solutions and spectral properties in Section 5.1, taking into account some connections with the theory of subordinacy.

Although analysis of the spectrum in terms of principal solutions is restricted to the non-oscillatory region $\left(-\infty, \lambda^{\star}\right)$, the method is still of interest because it uses properties of the solution space as a whole to identify the spectrum. This contrasts with the informal approach in terms of bounded solutions, which aims to locate the spectrum by applying a specific criterion (3) to the particular solution of (1) which satisfies (2), without any reference to the remainder of the solution space. It will be seen that the definition of a subordinate solution given in Section 3.1 directly extends the definition of a principal solution by replacing the pointwise comparison of solutions for large $r$ with limiting ratios of Hilbert space norms, thus enabling the idea of a principal solution to be applicable in both oscillatory and non-oscillatory regions. This key definition is fundamental 
to the theory of subordinacy, and enables precise correlations between the relative asymptotic behaviour of solutions of (1) and specific spectral properties of $H_{\alpha}$ to be established. The use of properties of the solution space as a whole is also a feature of related transfer matrix methods, which are particularly effective in connection with the absolutely continuous spectrum (see e.g. [30], [28], [17]) and will be briefly introduced in Section 5.1.

\section{The Method of Subordinacy}

For selfadjoint operators associated with (1), it is the behaviour of solutions at one or both endpoints of the interval $I$, not their intermediate properties, that determines the contribution to the spectrum at each fixed value of the spectral parameter $\lambda$. This situation is made precise by the theory of subordinacy, which provides rigorous criteria for locating minimal supports for the absolutely continuous and singular spectra, and also, in the case of two limit point endpoints, enables the simple and degenerate parts of the spectrum to be identified [15], [13], [14], [35]. Similar results have been shown to apply to related operators, such as the one-dimensional Dirac operator, the general Sturm-Liouville operator, infinite matrix operators and the random Schrödinger operator, and will be summarised in Section 4 .

The method of subordinacy is advantageous in several respects. In the first place, the results are independent of the detailed properties of $q$; only very general requirements, as, for example, that $q$ be locally integrable and that $L$ is in the limit point case at the infinite endpoints, need to be met. Moreover, a complete analysis of the spectrum can be achieved, at least in principle, by considering the behaviour of solutions at real values only of the spectral parameter $\lambda$. As a result, the considerable technicalities of the spectral function and Titchmarsh-Weyl $m$-function, which are key features in the derivation of the theory, can now be avoided in applications. Also, to identify the absolutely continuous component of $\sigma\left(H_{\alpha}\right)$, it is only necessary to consider the behaviour of solutions at the limit point endpoint(s), and for certain classes of potentials, the condition of non-subordinacy can be replaced by a much simpler boundedness criterion (see Section 5.1).

\subsection{The Half-Line Case}

We recall that in this case $L$ is assumed to be regular at $x=0$; the differential operator $H_{\alpha}$ acting on $\mathcal{H}=L_{2}([0, \infty))$ is then defined by

$$
H_{\alpha} f=L f, f \in \mathcal{D}\left(H_{\alpha}\right)
$$

where

$$
\mathcal{D}\left(H_{\alpha}\right)=\left\{f \in \mathcal{H}: L f \in \mathcal{H} ; f, f^{\prime} \text { locally a.c.; } \cos (\alpha) f(0)+\sin (\alpha) f^{\prime}(0)=0\right\}
$$


for some fixed $\alpha \in[0, \pi)$, and $q$ is locally integrable. Note that since $L$ is limit point at infinity, there is at most one solution of $L f=\lambda f$ in $\mathcal{H}$ for any $\lambda \epsilon$ $\mathbf{C}$, and that a boundary condition is needed only at 0 (see [15] and references therein).

Associated with $H_{\alpha}$ is a non-decreasing spectral function $\rho_{\alpha}: \mathbf{R} \rightarrow \mathbf{R}$ and it is convenient in the present context to define the spectrum, $\sigma\left(H_{\alpha}\right)$, to be the complement of those points of $\mathbf{R}$ in a neighbourhood of which $\rho_{\alpha}$ is constant. The spectral function generates a corresponding Borel-Stieltjes measure, $\mu_{\alpha}$, on $\mathbf{R}$ in the usual way, and the minimal supports of $\mu_{\alpha}$ provide an indication of where $\sigma\left(H_{\alpha}\right)$ is concentrated. Minimal supports (sometimes also known as essential supports) are defined as follows.

Definition 1. A subset $S$ of $\mathbf{R}$ is said to be a minimal support of a BorelStieltjes measure $\tau$ if

(i) $\tau(\mathbf{R} \backslash S)=0$,

(ii) whenever $S_{0} \subseteq S$ satisfies $\tau\left(S_{0}\right)=0$, then $\left|S_{0}\right|=0$, where $|\cdot|$

denotes Lebesgue measure.

It follows from the definition that minimal supports of $\mu_{\alpha}$ are unique up to Lebesgue and $\mu_{\alpha}$-null sets; while they may differ from $\sigma\left(H_{\alpha}\right)$ by sets of positive Lebesgue measure, there always exists a minimal support of $\mu_{\alpha}$ whose closure is the spectrum [15]. Since $\mu_{\alpha}$ can be decomposed uniquely into absolutely continuous, singular continuous and pure point parts, Definition 1 may also be applied to $\left(\mu_{\alpha}\right)_{\text {a.c. }},\left(\mu_{\alpha}\right)_{\text {s.c. }}$ and $\left(\mu_{\alpha}\right)_{p . p .}$.

The definition of a subordinate solution given below makes precise the concept of relative asymptotic smallness of a solution at a limit point endpoint and is meaningful even if all solutions are oscillatory or no solutions are in $L_{2}([0, \infty))$.

Definition 2. If $L$ is regular at 0 and in the limit point case at infinity, then a non-trivial solution $u_{s}(r, \lambda)$ of $L u=\lambda u$ is said to be subordinate at infinity if for every linearly independent solution $u(r, \lambda)$

$$
\lim _{N \rightarrow \infty} \frac{\left\|u_{s}(r, \lambda)\right\|_{N}}{\|u(r, \lambda)\|_{N}}=0
$$

where $\|\cdot\|_{N}$ denotes the $L_{2}([0, N])$ norm.

Note that subordinate solutions are unique up to multiplicative constants, and that if $(4)$ holds for one solution $u(r, \lambda)$ which is linearly independent from $u_{s}(r, \lambda)$, then it holds for every solution $u(r, \lambda)$ which is linearly independent from $u_{s}(r, \lambda)$.

The following theorem identifies precise correlations between the spectral parts of $H_{\alpha}$ and the asymptotics of solutions of $L u=\lambda u$, in terms of minimal supports of $\left(\mu_{\alpha}\right)_{a . c .},\left(\mu_{\alpha}\right)_{\text {s.c. }}$ and $\left(\mu_{\alpha}\right)_{\text {p.p. }}$. The derivation of this result is crucially dependent on the corresponding Titchmarsh-Weyl function $m_{\alpha}$, which is 
a Herglotz function on $\mathbf{C}^{+}$, and whose limiting behaviour as the real axis is approached normally is closely related both to a generalised derivative of $\mu_{\alpha}$, and to the subordinacy properties of solutions of (1) [2], [15].

Theorem 1 Minimal supports $\mathcal{M}_{\text {a.c. }}\left(H_{\alpha}\right), \mathcal{M}_{\text {s.c. }}\left(H_{\alpha}\right)$ and $\mathcal{M}_{\text {p.p. }}\left(H_{\alpha}\right)$ of $\left(\mu_{\alpha}\right)_{\text {a.c. }},\left(\mu_{\alpha}\right)_{\text {s.c. }}$ and $\left(\mu_{\alpha}\right)_{\text {p.p. }}$ respectively are as follows:

$$
\begin{aligned}
\mathcal{M}_{\text {a.c. }}\left(H_{\alpha}\right)= & \{\lambda \in \mathbf{R}: \text { no solution of } L u=\lambda u \text { is subordinate at infinity }\}, \\
\mathcal{M}_{\text {s.c. }}\left(H_{\alpha}\right)= & \{\lambda \in \mathbf{R}: \text { a solution of } L u=\lambda u \text { exists which satisfies the } \\
& \text { boundary condition at } 0, \text { is subordinate at infinity, but is } \\
& \text { not in } \left.L_{2}([0, \infty))\right\}, \\
\mathcal{M}_{\text {p.p. }}\left(H_{\alpha}\right)= & \left\{\lambda \in \mathbf{R}: \text { a non-trivial } L_{2}([0, \infty)) \text { solution of } L u=\lambda u\right. \\
& \text { exists which satisfies the boundary condition at } 0\} .
\end{aligned}
$$

Theorem 1 shows that there are striking distinctions between the asymptotic behaviour of solutions associated with the different parts of the spectrum. If $\lambda \in \mathcal{M}_{\text {a.c. }}\left(H_{\alpha}\right)$, then all solutions of $L u=\lambda u$ are, in some sense, of comparable asymptotic size at infinity, and this implies, by the limit point property, that no (non-trivial) solutions are in $L_{2}([0, \infty))$. The absence of an $L_{2}([0, \infty))$ solution is also a feature if $\lambda \in \mathcal{M}_{\text {s.c. }}\left(H_{\alpha}\right)$, although not, of course, when $\lambda \in \mathcal{M}_{\text {p.p. }}\left(H_{\alpha}\right)$.

Generally speaking, however, the more significant distinctions are between the singular and absolutely continuous supports of $\mu_{\alpha}$; thus, for example, if $\beta \neq \alpha(\bmod \pi)$ is a distinct boundary condition at 0 , it is immediate from the theorem that

$$
\mathcal{M}_{\text {s.c. }}\left(H_{\alpha}\right) \cap \mathcal{M}_{\text {s.c. }}\left(H_{\beta}\right)=\mathcal{M}_{p . p .}\left(H_{\alpha}\right) \cap \mathcal{M}_{p . p .}\left(H_{\beta}\right)=\emptyset,
$$

whereas

$$
\mathcal{M}_{\text {a.c. }}\left(H_{\alpha}\right)=\mathcal{M}_{\text {a.c. }}\left(H_{\beta}\right)
$$

which confirms well-known results of Kato and others concerning the stability of the absolutely continuous spectrum under finite rank perturbations [25].

It should be noted that some care is needed in interpreting the results of Theorem 1 , given the nature of the relationship between $\mathcal{M}_{\text {a.c. }}\left(H_{\alpha}\right), \mathcal{M}_{\text {s.c. }}\left(H_{\alpha}\right)$ and $\mathcal{M}_{p . p .}\left(H_{\alpha}\right)$ and the corresponding spectra, $\sigma_{a . c .}\left(H_{\alpha}\right), \sigma_{\text {s.c. }}\left(H_{\alpha}\right)$ and $\sigma_{p . p .}\left(H_{\alpha}\right)$, which are closed sets. For example, although it is true that $\sigma_{p . p .}\left(H_{\alpha}\right)=\emptyset$ if and only if $\mathcal{M}_{p . p .}\left(H_{\alpha}\right)=\emptyset$, analogous statements cannot be made for $\sigma_{a . c .}\left(H_{\alpha}\right)$ or $\sigma_{\text {s.c. }}\left(H_{\alpha}\right)$. Indeed we may have $\sigma_{\text {a.c. }}\left(H_{\alpha}\right)=\emptyset$ even if $\mathcal{M}_{\text {a.c. }}\left(H_{\alpha}\right) \neq \emptyset$, and similarly for $\sigma_{\text {s.c. }}\left(H_{\alpha}\right)$; however, the converse situation is not possible since, using well-known properties of absolutely continuous and singular continuous measures, it follows from $\sigma_{\text {a.c. }}\left(H_{\alpha}\right) \neq \emptyset$ that any minimal support of $\left(\mu_{\alpha}\right)_{\text {a.c. }}$ has positive Lebesgue measure, and from $\sigma_{\text {s.c. }}\left(H_{\alpha}\right) \neq \emptyset$ that any minimal support of $\left(\mu_{\alpha}\right)_{s}$. is an uncountable set of Lebesgue measure zero.

A similar situation holds in the full-line case, which we now consider. 


\subsection{The Full-Line Case}

Let $H$ denote the one-dimensional operator associated with (1) on $\mathcal{H}=L_{2}(\mathbf{R})$, let $q$ be locally integrable on $(-\infty, \infty)$ and suppose that $L$ is in the limit point case at both endpoints. Then the selfadjoint operator $H$ is uniquely defined by

$$
H f=L f, f \in \mathcal{D}(H),
$$

where

$$
\mathcal{D}(H)=\left\{f \in \mathcal{H}: L f \in \mathcal{H} ; f, f^{\prime} \text { locally a.c. }\right\} .
$$

The analogue of the spectral function is now a $2 \times 2$ positive semidefinite spectral matrix function $\left(\rho_{i j}\right)$, and a suitable spectral measure, the so-called trace measure, $\mu$, is generated from the sum of its diagonal terms.

Let $H_{0}^{-}, H_{0}^{+}$respectively denote the selfadjoint operators on $L_{2}((-\infty, 0])$ and $L_{2}([0, \infty))$, which are defined in the usual way by $L$, together with a Dirichlet boundary condition at 0 . In the derivation of minimal supports for the fullline operator $H$, a delicate relationship between the trace measure $\mu$ and the Titchmarsh-Weyl $m$-functions associated with $H_{0}^{-}$and $H_{0}^{+}$is identified, which is then combined with application of Theorem 1 to $H_{0}^{-}$and $H_{0}^{+}$, to give the following theorem [13]; note that the definition of a solution which is subordinate at $-\infty$ is entirely analogous to that of Definition 2, except that the $L_{2}([0, N])$ norm is now replaced by the $L_{2}([-N, 0])$ norm.

Theorem 2 Minimal supports $\mathcal{M}_{\text {a.c. }}(H), \mathcal{M}_{\text {s.c. }}(H)$ and $\mathcal{M}_{\text {p.p. }}(H)$ of $\mu_{\text {a.c. }}$, $\mu_{\text {s.c. }}$ and $\mu_{\text {p.p. }}$ respectively are as follows:

$$
\begin{aligned}
\mathcal{M}_{\text {a.c. }}(H)= & \{\lambda \in \mathbf{R}: \text { no solution of } L u=\lambda u \text { is subordinate at }-\infty\} \\
& \cup\{\lambda \in \mathbf{R}: \text { no solution of } L u=\lambda u \text { is subordinate at }+\infty\}, \\
\mathcal{M}_{\text {s.c. }}(H)= & \{\lambda \in \mathbf{R}: \text { a solution of } L u=\lambda u \text { exists which is subordinate } \\
& \text { both at } \left.+\infty \text { and at }-\infty, \text { but is not in } L_{2}(\mathbf{R})\right\}, \\
\mathcal{M}_{\text {p.p. }}(H)= & \left\{\lambda \in \mathbf{R}: \text { a non-trivial } L_{2}(\mathbf{R}) \text { solution of } L u=\lambda u \text { exists }\right\} .
\end{aligned}
$$

Using Theorems 1 and 2, the well-known result that

$$
\sigma_{\text {a.c. }}(H)=\sigma_{\text {a.c. }}\left(H_{0}^{-}\right) \cup \sigma_{\text {a.c. }}\left(H_{0}^{+}\right)
$$

follows easily from the fact that

$$
\mathcal{M}_{\text {a.c. }}(H)=\mathcal{M}_{\text {a.c. }}\left(H_{0}^{-}\right) \cup \mathcal{M}_{\text {a.c. }}\left(H_{0}^{+}\right) .
$$

The analogous situation for the singular spectrum is less straightforward, since the matching of subordinate solutions at the decomposition point 0 is involved; taking this into account, we obtain, with obvious notation,

$$
\mathcal{M}_{s .}(H)=\cup_{\alpha}\left(\mathcal{M}_{s .}\left(H_{\alpha}^{-}\right) \cap \mathcal{M}_{s .}\left(H_{\alpha}^{+}\right)\right)
$$


where $\mathcal{M}_{\text {s. }}(H)=\mathcal{M}_{\text {s.c. }}(H) \cup \mathcal{M}_{p . p .}(H)$, and the union is taken over all $\alpha \in$ $[0, \pi)$.

An important issue in the full-line case is that of spectral multiplicity, which may be 1 or 2 ; in the half-line case, the question effectively does not arise, since the spectrum is always simple. A significant contribution to this topic is due to I.S. Kac, who identified necessary and sufficient conditions for the existence of degenerate spectrum in terms of the boundary behaviour of the $m$-functions associated with $H_{\alpha}^{-}$and $H_{\alpha}^{+}$[24]. By combining Kac's result with Theorem 2, we obtain the following result [14].

Theorem $3 H$ has spectral multiplicity 2 if and only if the Lebesgue measure of the set

$$
\begin{aligned}
\mathcal{M}_{2}(H)= & \{\lambda \in \mathbf{R}: \text { no solution of } L u=\lambda u \text { is subordinate at }-\infty\} \\
& \cap\{\lambda \in \mathbf{R}: \text { no solution of } L u=\lambda u \text { is subordinate at }+\infty\}
\end{aligned}
$$

is strictly positive; otherwise the spectrum of $H$ is simple.

Thus the degenerate spectrum of $H$, if it exists, is effectively concentrated on $\mathcal{M}_{2}(H)$, which is a subset of $\mathcal{M}_{\text {a.c. }}(H)$, and the simple spectrum on $\mathcal{M}_{1}(H)=$ $\mathcal{M}(H) \backslash \mathcal{M}_{2}(H)$, where $\mathcal{M}(H)=\mathcal{M}_{\text {a.c. }}(H) \cup \mathcal{M}_{\text {s. }}(H)$ is a minimal support of $\mu$; for further details, see [14]. Note that $\mathcal{M}_{2}(H)$ in Theorem 3 cannot be replaced by $S=\sigma_{\text {a.c. }}\left(H_{0}^{-}\right) \cap \sigma_{\text {a.c. }}\left(H_{0}^{+}\right)$, since it is known that operators $H$ exist for which $|S|>0$, but $\left|\mathcal{M}_{2}(H)\right|=\emptyset$ (see e.g. [14, Example 6.5]).

It is interesting to observe from Theorem 3 that the simple part of $\sigma(H)$ is characterised by the existence of a relatively small solution of $L u=\lambda u$ on $\mathbf{R}$; this smallness need only be strict at one of the endpoints. The degenerate spectrum, on the other hand, is characterised by all solutions of $L u=\lambda u$ on $\mathbf{R}$ being indistinguishable in terms of relative asymptotic size. On $\mathbf{R} \backslash \mathcal{M}(H)$, which includes the resolvent set, the solutions of $L u=\lambda u$ are not well-ordered in the sense that, although there exists a solution, $u_{-\infty}$, which is subordinate at $-\infty$, and a solution, $u_{+\infty}$, which is subordinate at $+\infty$, these solutions are linearly independent; thus there is no non-trivial solution of $L u=\lambda u$ which is relatively small at both endpoints.

\section{Extensions and Generalisations}

The usefulness of the theory of subordinacy in a range of spectral problems has led to a number of extensions to related operators. In each of the cases which we now describe, a limit point, limit circle theory is known, which is analogous to the Weyl theory for the standard singular Sturm-Liouville case. We assume therefore, as before, that 0 is a regular endpoint in the half-line case and that the infinite endpoints are limit point.

Generalised Sturm-Liouville operators 
Consider the generalised Sturm-Liouville operator $H_{\alpha}$ which is associated with the differential equation

$$
L u:=-\left(p u^{\prime}\right)^{\prime}(r)+q(r) u(r)=\lambda w(r) u(r), \quad r \in[0, \infty),
$$

and boundary condition $\cos (\alpha) u(0)+\sin (\alpha)\left(p u^{\prime}\right)(0)=0$ on the weighted Hilbert space $L_{2}^{w}([0, \infty))$. Here $\alpha \in[0, \pi)$ is fixed and $p, q, w$ are real valued functions with $p, w>0$ and $p^{-1}, q, w$ locally integrable. Then Theorem 1 holds with $\|\cdot\|_{N}=\left(\int_{0}^{N}|\cdot|^{2} w(r) d r\right)^{\frac{1}{2}}[8]$.

\section{Separated Dirac operators}

For spherically symmetric potentials, the separated Dirac equation may be written in system form

$$
\left(\begin{array}{rr}
0 & -1 \\
1 & 0
\end{array}\right) \underline{u^{\prime}}(r)+\left(\begin{array}{ll}
q_{1}(r) & q_{2}(r) \\
q_{2}(r) & q_{3}(r)
\end{array}\right) \underline{u}(r)=\lambda \underline{u}(r), \quad r \in[0, \infty),
$$

where $q_{1}, q_{2}, q_{3}$ are real valued, locally integrable functions, and $\underline{u}(r)=\left(u_{1}, u_{2}\right)^{t}$. To construct the corresponding selfadjoint operator $H_{\alpha}$, it is necessary to impose an initial condition of the form $\cos (\alpha) u_{1}(0)+\sin (\alpha) u_{2}(0)=0$, for some $\alpha \in$ $[0, \pi)$. Then Theorem 1 holds for $H_{\alpha}$ under the usual $L_{2}([0, \infty)) \otimes \mathbf{C}^{2}$ norm, and Theorem 2 is also valid for the corresponding operator $H$ on $L_{2}(\mathbf{R}) \otimes \mathbf{C}^{2}$ [3].

\section{Infinite matrix operators}

Let $H_{\alpha}$ be the selfadjoint operator associated with the semi-infinite matrix

$$
\left(\begin{array}{cccccc}
a_{0} & b_{0} & & & & \\
b_{0} & a_{1} & b_{1} & & 0 & \\
& b_{1} & a_{2} & b_{2} & & \\
& & b_{2} & a_{3} & b_{3} & \\
& 0 & & \ldots & \ldots & \ldots \\
& & & & \ldots & \ldots
\end{array}\right)\left(\begin{array}{c}
u_{0} \\
u_{1} \\
u_{2} \\
u_{3} \\
\ldots \\
\ldots
\end{array}\right)=\lambda\left(\begin{array}{c}
w_{0} u_{0} \\
w_{1} u_{1} \\
w_{2} u_{2} \\
w_{3} u_{3} \\
\ldots \\
\ldots
\end{array}\right)
$$

and boundary condition $\cos (\alpha) u_{1}+\sin (\alpha) b_{0}\left(u_{1}-u_{0}\right)=0$, where $\alpha \in[0, \pi)$ is fixed, and $a_{i}, b_{i}, w_{i} \in \mathbf{R}, b_{i} \neq 0, w_{i}>0$, for $i=0,1,2, \ldots \ldots$. This case includes the Jacobi matrix operator for which $b_{i}=w_{i}=1$, and Theorem 1 holds for $H_{\alpha}$ under the usual $\ell_{2}^{w}$ norm with $\|\underline{u}\|_{N}=\left(\sum_{n=0}^{N} w_{n}\left|u_{n}\right|^{2}\right)^{\frac{1}{2}}$ [7], [26], [45].

An interesting variant is the matrix operator associated with orthogonal polynomials on the unit circle and the Szegó recurrence relations,

$\vec{X}(z, n)=\frac{1}{\left(1-\left|a_{n}\right|^{2}\right)^{\frac{1}{2}}}\left(\begin{array}{cc}z & a_{n} \\ \overline{a_{n}} z & 1\end{array}\right) \vec{X}(z, n-1), n \in \mathbf{N},\left|a_{n}\right|<1,|z| \in[0,1]$,

with initial condition $\vec{X}(z, 0)=(1,1)^{t}$. Here the interior of the unit circle takes the place of $\mathbf{C}^{+}$in the construction of an analogue, $F(z)$, of the 
Titchmarsh-Weyl $m$-function, and $F(z)$ in turn is associated with an orthogonality measure $\mu$ on the unit circle which has a similar role to the spectral measure $\mu_{\alpha}$ on $\mathbf{R}$ in the half-line case. Theorem 1 now holds for $\mu$ with $\|\vec{X}(z)\|_{N}=\left(\sum_{n=0}^{N}\|\vec{X}(z, n)\|^{2}\right)^{\frac{1}{2}}$, where $\vec{X}(z)=(\vec{X}(z, 0), \ldots, \vec{X}(z, n), \ldots)$, $\vec{X}(z, n)=\left(x_{1}(z, n), x_{2}(z, n)\right)^{t}$ and $\|\vec{X}(z, n)\|=\left(\left|x_{1}(z, n)\right|^{2}+\left|x_{2}(z, n)\right|^{2}\right)^{\frac{1}{2}}$ $[17]$.

\section{Random Schrödinger operators}

Here the general form of the differential operator considered is

$$
L_{w} u:=-u^{\prime \prime}(r)+q_{w}(r) u(r)=\lambda u(r), \quad r \in[0, \infty),
$$

where $\left\{q_{w}(r): r \in[0, \infty)\right\}$ is a random function on a probability space $(\Omega, \mathcal{F}, P)$, or a random generalised function. In this context, we assume that $L_{w}$ is in the limit point case for $P$-almost all $w$, so that $H_{w, \alpha}$, defined from $L_{w}$ together with the boundary condition $\cos (\alpha) u(0)+\sin (\alpha) u^{\prime}(0)=0$, is selfadjoint with probability 1 ; the unique selfadjoint operator $H_{w}$ is defined by $L_{w}$ on $\mathbf{R}$ in a similar way. Then under the assumption that a technical condition related to the well known Kotani trick holds, analogous results to Theorems 1 and 2 can be established for $\lambda$-intervals of the real line [32].

Remark In addition to extensions of the theory to a number of related operators, some refinements to the concept of a subordinate solution have been proposed and used in applications; for example, power law subordinacy [23], strong non-subordinacy [8], uniform non-subordinacy [49] and sequential subordinacy [35], [44]. Power law subordinacy is a significant generalisation of the original concept, which enables dimensional Hausdorff properties of the singular continuous spectrum to be investigated, and has provided detailed results for the almost Mathieu operator and Fibonacci Hamiltonian. Strong and uniform nonsubordinacy are associated with the relatively "well-behaved" situation where no solution of (1) is subordinate for any $\lambda$ in a compact interval $I$; in such cases, various uniformity properties on $I$ can be inferred for the ratios of norms of solutions and for the spectral density functions $\rho_{\alpha}^{\prime}$. From Definition 2 it is evident that, to establish non-subordinacy of solutions, it is only necessary (and usually much more convenient) to demonstrate non-sequential subordinacy for a single sequence.

\section{Applications}

In principle, Theorems 1, 2, 3 and their extensions provide distinguishing criteria which enable a complete and detailed analysis of the spectrum to be carried out for a range of differential and difference operators. However, in practice it is rare for explicit expressions for the solutions of the governing equations to be 
available, so that in applications the theory is often used indirectly or in conjunction with suitable asymptotic estimates of the behaviour of the solutions at the endpoints. Some typical strategies include use of the Liouville-Green approximation, application of Levinson's theorem and its extensions, various results on boundedness and non-subordinacy, and related transfer matrix methods.

Before considering specific examples, we briefly return to the relationship between bounded solutions and spectral properties.

\subsection{Boundedness Revisited}

The following result, linking boundedness of solutions to non-subordinacy, can greatly simplify the analysis when absolutely continuous spectrum is present [44].

Lemma 1 Let $L$ be as in (1) with $I=[0, \infty)$, and suppose that

$$
\sup _{x \geq 0} \int_{x}^{x+1} q_{-}(r) d r<\infty
$$

where $q_{-}$denotes the negative part of $q$. Then if all solutions of $L u=\lambda u$ are bounded for some fixed $\lambda \in \mathbf{R}, L$ is in the limit point case at infinity and, for the same fixed $\lambda$,

(i) $u^{\prime}$ is bounded for all solutions $u$ of $L u=\lambda u$,

(ii) no solution of $L u=\lambda u$ is subordinate at infinity.

Note that Lemma 1 shows that, provided (5) is satisfied, the boundedness of solutions of $L u=\lambda u$ is a sufficient condition for $\lambda$ to be in $\mathcal{M}_{a . c .}\left(H_{\alpha}\right)$; however, it is not a necessary condition, as may be seen by considering a fundamental set of solutions, $\left\{(r+1)^{\frac{1}{2}} \cos \left(\frac{1}{2} \ln (r+1)\right),(r+1)^{\frac{1}{2}} \sin \left(\frac{1}{2} \ln (r+1)\right)\right\}$, of $L u=\lambda u$ when $q(r)=-2^{-1}(r+1)^{-2}$ and $\lambda=0$. Variants of Lemma 1 for the Dirac and Jacobi matrix operators may be found in [4], [40], [45], and an alternative proof is contained in [41].

It turns out that Lemma 1 may be recast in terms of transfer matrices. To see this, let $\lambda \in \mathbf{R}$ be fixed and suppose that for $x \in I=[0, \infty),\left\{u_{\lambda}(x), v_{\lambda}(x)\right\}$ is a fundamental set of solutions of $L u=\lambda u$ satisfying $u_{\lambda}(0)=v_{\lambda}^{\prime}(0)=0$, $u_{\lambda}^{\prime}(0)=v_{\lambda}(0)=1$.

Then setting

$$
T_{\lambda}(x):=\left(\begin{array}{ll}
u_{\lambda}^{\prime}(x) & v_{\lambda}^{\prime}(x) \\
u_{\lambda}(x) & v_{\lambda}(x)
\end{array}\right)
$$

it is easy to check that for any solution $y_{\lambda}(x)=a u_{\lambda}(x)+b v_{\lambda}(x)$ of $L u=\lambda u$, with $a, b \in \mathbf{C}$,

$$
T_{\lambda}(x)\left(\begin{array}{c}
y_{\lambda}^{\prime}(0) \\
y_{\lambda}(0)
\end{array}\right)=\left(\begin{array}{c}
y_{\lambda}^{\prime}(x) \\
y_{\lambda}(x)
\end{array}\right)
$$


If we now define

$$
\left\|T_{\lambda}(x)\right\|:=\sup _{\|y\|=1}\left\|T_{\lambda}(x) y\right\|=\sup _{|a|^{2}+|b|^{2}=1}\left(\left|y_{\lambda}^{\prime}(x)\right|^{2}+\left|y_{\lambda}(x)\right|^{2}\right)^{\frac{1}{2}},
$$

where $y=\left(y_{\lambda}^{\prime}, y_{\lambda}\right)^{t}$, then it may be seen from Lemma 1 that, subject to (5), all solutions of $L u=\lambda u$ are bounded if and only if $\lim _{\sup } \rightarrow \infty\left\|T_{\lambda}(x)\right\|<$ $\infty$. It follows that issues relating to boundedness of solutions and spectral properties can be investigated using results from subordinacy and from the study of transfer matrices. Interest in the latter approach has already led to a number of promising new developments (see e.g. [17], [30], [28]).

\subsection{Examples}

In each of the following examples, it is readily verified that, as appropriate, 0 is a regular endpoint and the infinite endpoints are limit point. The operators $H_{\alpha}$ and $H$ are defined as in Sections 2 and 3 unless otherwise stated.

Example 1 Let $q=p+s+w$ on $[0, \infty)$, where $p \in L_{1}([0, \infty)), s$ is smooth and long range with $s^{\prime} \in L_{1}([0, \infty))$, and the von Neumann-Wigner part, $w$, satisfies $w(r) \rightarrow 0$ as $r \rightarrow \infty$, and is conditionally integrable, i.e. $\lim _{r \rightarrow \infty} \int_{0}^{r} w(r) d r$ exists. Then for all $\alpha \in[0, \pi), \sigma\left(H_{\alpha}\right)$ is purely absolutely continuous on $(0, \infty)$ apart from at most a countable set of embedded eigenvalues, known as resonances. This result is obtained by using asymptotic integration to obtain suitable estimates of solutions of $L u=\lambda u$ for $\lambda>0$ and large $r$, and applying Theorem 1 [3]; some generalisations are obtained in [4], where Lemma 1 and a limiting absorption principle are also used.

Example 2 Let $L_{0}:=-\frac{d^{2}}{d r^{2}}+\cos (r), \quad r \in(-\infty, \infty)$,

$$
L:=-\frac{d^{2}}{d r^{2}}+\cos (r)+\delta \cos \left(|r|^{\gamma}\right), \quad 0<\gamma<1, \quad r \in(-\infty, \infty),
$$

and denote by $\left(a_{n}, b_{n}\right), n=1,2, \ldots$. , the stability intervals of the selfadjoint operator $H_{0}$ associated with $L_{0}$. Then it is well known that $\sigma\left(H_{0}\right)=\cup_{n}\left[a_{n}, b_{n}\right]$, and is purely absolutely continuous, with $b_{n}<a_{n+1}$ for all $n \in \mathbf{N}$; moreover, as $n \rightarrow \infty$, the length of the stability intervals is $O(n)$ and the length of the gaps becomes arbitrarily small (see e.g. [11], [36, Chapter XIII, Section 16, Example 1]). However, the essential spectrum of the perturbed operator $H$ associated with $L$ is a countable union of closed intervals, each consisting of a central band of absolutely continuous spectrum and two outer bands of singular spectrum, these banded intervals being disjoint for suitably chosen $\delta$ and sufficiently small $n$. In addition, every interval of absolutely continuous spectrum, respectively gap in the essential spectrum, of the perturbed operator $H$ is a subset of a stability interval, respectively subset of a spectral gap, of the unperturbed operator $H_{0}$. To achieve these results, trace class methods are used to show that if $(b, a)$ 
is a spectral gap of $H_{0}$, then $(b-\delta, a+\delta) \cap \sigma_{a . c .}(H)=\emptyset$, and adaptations of arguments used in [45] are combined with Lemma 1 and Theorems 1 and 2 to show that if $(a, b)$ is a stability interval of $H_{0}$, then $(a+\delta, b-\delta)$ is a stability interval of $H$ [46]. The degeneracy of $\sigma_{\text {a.c. }}(H)$ can be inferred from Theorem 3.

Example 3 Using the notation of Section 4, let $H_{w}$ denote the GoldsheidMolchanov-Pastur model and $H_{w, \alpha}$ a selfadjoint operator arising from $L_{w}:=$ $-d^{2} / d r^{2}+k W(r), r \in[0, \infty)$, where $k>0$ and $W(r)$ is Gaussian white noise. If $F \geq 0$ is the intensity of a constant electric field, then with probability 1 the perturbed GMP operator $H_{w}^{F}=H_{w}-F r$ has purely absolutely continuous spectrum with $\sigma\left(H_{w}^{F}\right)=\mathbf{R}$, and the perturbed operator $H_{w, \alpha}^{F}=H_{w, \alpha}-F r$ has dense pure point spectrum on $\mathbf{R}$ for $F<k^{2} / 2$ and purely singular continuous spectrum with $\sigma\left(H_{w, \alpha}^{F}\right)=\mathbf{R}$ for $F \geq k^{2} / 2$. These results are established by using the asymptotic expansions of Airy functions in a transformation of the eigenvalue equations and applying analogues of Theorems 1 and 2 for random operators [32].

Example 4 Let $L$ be as in (1), with $I=[0, \infty)$ and $q: I \rightarrow \mathbf{C}$ satisfying $\operatorname{Im} q \geq 0$ and $\operatorname{Im} q(r) \rightarrow 0$ as $r \rightarrow \infty$. Let $H_{\alpha}$ denote the non-selfadjoint operator associated with $L$ and a selfadjoint boundary condition at the origin (cf. $(2))$. Then if $\operatorname{Im} q \notin L_{1}([0, \infty))$, the absolutely continuous spectrum of $H_{\alpha}$ is empty. This result depends on a characterisation in terms of Hardy spaces of the absolutely continuous subspace, $N_{e}$, of a maximally dissipative, completely non-selfadjoint operator with essential spectrum on the real axis (see e.g. [33]). Also involved are an analogue of the Titchmarsh-Weyl function and an associated spectral measure, which together enable a theory of subordinacy to be established for the class of operators considered, through which the proof is completed [37].

Acknowledgements I should like to express my gratitude to Professor Amrein and the organising committee for inviting me to contribute to the Sturm Bicentennial Conference. I also wish to thank the referees of this paper for their careful reading of the manuscript and constructive suggestions.

\section{References}

[1] W. O. Amrein and V. Georgescu, Strong asymptotic completeness of wave operators for highly singular potentials, Helv. Phys. Acta 47 (1974), 517 533.

[2] N. Aronszajn, On a problem of Weyl in the theory of singular SturmLiouville equations, Amer. J. Math. 79, (1957), 597 - 610.

[3] H. Behncke, Absolute continuity of Hamiltonians with von NeumannWigner potentials, Proc. Amer. Math. Soc. 111 (1991), 373 - 384. 
[4] H. Behncke, Absolute continuity of Hamiltonians with von NeumannWigner potentials II, Manuscripta Math. 71 (1991), 163 - 181.

[5] M. Bôcher, Leçons sur les Méthodes de Sturm, Gauthier-Villars, Paris, 1917.

[6] R. Carmona, One-dimensional Schrödinger operators with random or deterministic potentials: New spectral types, J. Funct. Anal. 51 (1983), 229 -258 .

[7] S. L. Clark, A spectral analysis for selfadjoint operators generated by a class of second order difference equations, J. Math. Anal. Appl. 197 (1996), 267 -285 .

[8] S. L. Clark and D. B. Hinton, Strong nonsubordinacy and absolutely continuous spectra for Sturm-Liouville equations, Differential Integral Equations 6 (1993), 573 - 586.

[9] R. del Rio, S. Jitomirskaya, N. Makarov and B. Simon, Singular continuous spectrum is generic, Bull. Amer. Math. Soc. 31 (1994), 208 - 212.

[10] P. A. M. Dirac, Principles of Quantum Mechanics, 4th Edition, Oxford University Press, 1957.

[11] P. Djakov and B. Mityagin, Spectral gaps of the periodic Schrödinger operator when its potential is an entire function, Adv. in Appl. Math. 31 (2003), $562-596$.

[12] W. N. Everitt, Charles Sturm and the development of Sturm-Liouville theory in the years 1900 to 1950, Proceedings of the Sturm Bicentennial Conference, University of Geneva, 2003.

[13] D. J. Gilbert, On subordinacy and analysis of the spectrum of Schrödinger operators with two singular endpoints, Proc. Royal Soc. Edinburgh Sect. A 112 (1989), 213 - 229.

[14] D. J. Gilbert, On subordinacy and spectral multiplicity for a class of singular differential operators, Proc. Royal Soc. Edinburgh Sect. A 128 (1998), $549-584$.

[15] D. J. Gilbert and D. B. Pearson, On subordinacy and analysis of the spectrum of one-dimensional Schrödinger operators, J. Math. Anal. Appl. 128 (1987), $30-56$.

[16] I. M. Glazman, Direct Methods of Qualitative Spectral Analysis of Singular Differential Operators, Israel Program for Scientific Trans., Jerusalem, 1965. 
[17] L. Golinskii and P. Nevai, Szegő difference equations, transfer matrices and orthogonal polynomials on the unit circle, Comm. Math, Phys. 223 (2001), $223-259$.

[18] P. Hartman, A characterisation of the spectra of the one-dimensional wave equations, Amer. J. Math. 71 (1949), 915 - 920.

[19] P. Hartman and A. Wintner, An oscillation theorem for continuous spectra, Proc. Nat. Acad. Sci. U.S.A. 33 (1947), 376 - 379.

[20] P. Hartman and A. Wintner, Oscillatory and non-oscillatory linear differential equations, Amer. J. Math. 71 (1949), 627 - 649.

[21] Don Hinton, Sturm's 1836 oscillation results: Evolution of the theory, Proceedings of the Sturm Bicentennial Conference, University of Geneva, 2003.

[22] A. Hinz and G. Stolz, Polynomial boundedness of eigensolutions and the spectrum of Schrödinger operators, Math. Ann. 294 (1992), 195 - 211.

[23] S. Jitomirskaya and Y. Last, Power-law subordinacy and singular spectra I: Half-line operators, Acta Math. 183 (1999), 171 - 179; II: Line operators Comm. Math. Phys. 211 (2000), 643 - 658.

[24] I. S. Kac, On the multiplicity of the spectrum of a second order differential operator, Soviet Math. Doklady 3 (1962), 1035 - 1039; On the multiplicity of the spectrum of a second order differential operator and the associated eigenfunction expansion (Russian), Izv. Akad. Nauk. SSSR, Ser. Mat. 27 (1963), 1081 - 1112.

[25] T. Kato, On finite dimensional perturbations of selfadjoint operators, $J$. Math. Soc. Japan 9 (1957), 239 - 249.

[26] S. Khan and D. B. Pearson, Subordinacy and spectral theory for infinite matrices, Helv. Phys. Acta 65 (1992), 505 - 527.

[27] A. Kiselev, Absolutely continuous spectrum of one-dimensional Schrödinger operators and Jacobi matrices with slowly decreasing potentials, Comm. Math. Phys. 79 (1996), 377 - 400.

[28] A. Kiselev, Y. Last and B. Simon, Modified Prüfer transforms and absolutely continuous spectrum of one-dimensional Schrödinger operators, Comm. Math. Phys. 194 (1998), 1 - 45.

[29] Y. Last, Quantum dynamics and decompositions of singular continuous spectra, J. Funct. Anal. 142 (1996), 406 - 445.

[30] Y. Last and B. Simon, Eigenfunctions, transfer matrices and absolutely continuous spectrum of one-dimensional Schrödinger operators, Invent. Math. 135 (1999), 329 - 367. 
[31] J. Liouville and C. Sturm, Extrait d'un mémoire sur le développment des fonctions en série dont les différents termes sont assujettis à satisfaire une même équation différentielle linéaire, contenant un paramètre variable, $J$. Math. Pures Appl. 2 (1837), 220 - 223.

[32] N. Minami, Random Schrödinger operators with a constant electric field, Ann. Inst. H. Poincaré, Phys. Théor. 56 (1992), 307 - 344.

[33] S. N. Naboko, A functional model of perturbation theory and its applications to scattering theory (Russian), Trudy Mat. Inst. Steklov 147 (1980), 86 - 114; English transl. in Proc. Steklov Inst. Math. 2 (1981), 85 - 116.

[34] D. B. Pearson, Singular continuous measures in scattering theory, Comm. Math. Phys. 60 (1978), 13 - 36.

[35] D. B. Pearson, Quantum Scattering and Spectral Theory, Academic Press, 1988.

[36] M. Reed and B. Simon, Methods of Modern Mathematical Physics, Vol. IV: Analysis of Operators, Academic Press, London, 1978.

[37] R. Romanov, On instability of the absolutely continuous spectrum of dissipative Schrödinger operators and Jacobi matrices, preprint available from roma@RR1750.spb.edu.

[38] L. I. Schiff, Quantum Mechanics, McGraw-Hill, New York, 1949.

[39] E. Schrödinger, Quantisierung als Eigenwertproblem, Ann. Physik 80 (1926), $437-490$.

[40] B. Simon, Schrödinger semigroups, Bull. Amer. Math. Soc. 7 (1982), 447 526 .

[41] B. Simon, Bounded eigenfunctions and absolutely continuous spectrum for one dimensional Schrödinger operators, Proc. Amer. Math. Soc. 124 (1996), $3361-3369$.

[42] B. Simon, Sturm oscillation and comparison theorems, Proceedings of the Sturm Bicentennial Conference, University of Geneva, 2003.

[43] B. Simon and G. Stolz, Operators with singular continuous spectrum V. Sparse potentials, Proc. Amer. Math. Soc. 124 (1996), 2073 - 2080.

[44] G. Stolz, Bounded solutions and absolute continuity of Sturm-Liouville operators, J. Math. Anal. Appl. 169 (1992), 210 - 228.

[45] G. Stolz, Spectral theory for slowly oscillating potentials, I. Jacobi matrices, Manuscripta Math. 84 (1994), 245 - 260. 
[46] G. Stolz, Spectral theory for slowly oscillating potentials, II. Schrödinger operators, Math. Nachr. 183 (1997), 275 -294.

[47] C. Sturm, Sur les équations différentielles linéaires du second ordre, $J$. Math. Pures Appl. 1 (1836), 106 - 186.

[48] J. Weidmann, Spectral Theory of Ordinary Differential Operators, Lecture Notes in Mathematics 1258, Springer-Verlag, Berlin, 1987.

[49] J. Weidmann, Uniform nonsubordinacy and the absolutely continuous spectrum, Analysis 16 (1996), 89 - 99.

[50] J. Weidmann, Spectral theory of Sturm-Liouville operators: Approximation by regular problems, Proceedings of the Sturm Bicentennial Conference, University of Geneva, 2003.

[51] H. Weyl, Über gewöhnliche Differentialgleichungen mit Singularitäten und die zugehörigen Entwicklungen willkürlicher Funktionen, Math. Ann. 68 (1910), 220 - 269.

[52] A. Wintner, On the smallness of isolated eigenfunctions, Amer. J. Math. 71 (1949), 603 - 611.

[53] S. Ya. Zhitomirskaya, Singular spectral properties of a one-dimensional Schrödinger operator with almost periodic potential, Adv. Soviet Math. 3 (1991), 215 - 254. 